\title{
Design of a hydrogen-powered bicycle for sustainable mobility
}

\author{
Paolo Di Giorgio ${ }^{1,2^{*}}$, Giovanni Di Ilio ${ }^{1,2}$, Gabriele Scarpati ${ }^{1,2}$, Giovanni Erme $^{2,3}$, Elio Simeoni ${ }^{1,2}$, Elio Jannelli ${ }^{1,2}$ \\ ${ }^{1}$ University of Naples "Parthenope", Naples, Italy \\ ${ }^{2}$ ATENA Future Technology, Naples, Italy \\ ${ }^{3}$ University of Cassino and Southern Lazio, Cassino, Italy
}

\begin{abstract}
Hydrogen-powered vehicles are emerging as a key source for a clean and sustainable mobility scenario. In particular, hydrogen technologies have a great potential for light mobility in urban areas, where traffic congestion may cause very high levels of local pollution. In this context, hybrid fuel cell/battery vehicles represent a promising solution, since they allow for extended driving range and short recharge time, which are two of the major concerns related to electric propulsion, in general. In this work, a new plug-in fuel cell electric bicycle concept is presented, where the on-board energy storage is realized by means of an innovative system integrating a battery pack with a metal hydride hydrogen tank. This solution allows to achieve very high performance in terms of riding range, which are unattainable with traditional battery electric bicycles. In particular, the hybrid energy storage system is conceived to provide an optimal thermal management of the two integrated components. The proposed design is developed on the basis of typical duty cycles acquired during on-road measurements. A prototype of the bicycle is then realized and bench-tested in order to assess design consistency and to evaluate its performances. The results show that the riding range of the new hydrogen-fuelled bicycle is about three times higher than the one for a similar electric bicycle.
\end{abstract}

\section{Introduction}

Hydrogen is capturing an unprecedented attention in industry as a versatile and sustainable energy carrier for a zero-emissions mobility scenario, and it is nowadays very much part of the political agenda of several Countries. Fuel Cell Electric Vehicles (FCEVs) are progressively taking hold in the market, as a viable alternative to Battery Electric Vehicles (BEVs). In particular, Plug-in Fuel Cell Electric Vehicles (PFCEVs) represents possibly an even more appealing solution than FCEVs, since they are generally more energy efficient, due to the possibility of component downsizing and optimization of control strategies $[1,2]$. In addition, the plug-in fuel cell/battery hybrid powertrain configuration is suitable for implementation across a wide range of road vehicle sizes and typologies, from heavy duty trucks [3-5] to lightweight vehicles such as bicycles, scooters and threewheelers [6-8]. One crucial aspect related to PFCEVs concerns the on-board hydrogen storage. Typically, this is realized by means of a compressed gas system, given the relatively high energy density features of this technology. However, other common solutions involve the use of hydrogen carriers, like ammonia $[9,10]$, or the adoption of metal hydrides for solid-state storage of hydrogen [11]. In particular, despite the growing interest in developing cleaner and more efficient solutions for light-mobility, the potential of hydrogen technologies for this class of vehicles has not been fully exploited yet, and only a very limited number of prototypes is available.

In this work, the authors propose the design of the power unit and of an innovative thermally integrated energy storage system for a plug-in fuel cell electric bike, hereafter referred to HyBike. In particular, the proposed energy storage solution consists of a small sized battery pack partially integrated into a $\mathrm{MH}$ tank for hydrogen storage. In this way, the waste heat of the battery pack can be effectively transferred via conduction to the $\mathrm{MH}$ tank, thus promoting hydrogen desorption and enabling a suitable thermal management of the battery pack. Moreover, the realized energy storage system has enhanced energy density, which results in an increase of about three times the riding range of the vehicle. The proposed design for the HyBike power unit and storage system is developed on the basis of power profiles acquired during road-tests for the original electric bicycle (e-bike) from which the new HyBike originates, and that are representative of typical operations. A prototype for the designed components is then realized and benchtested using a Battery Testing System (BTS) reproducing the acquired operational profiles. The aim of the experimental tests was to assess the correct operation of the power unit and to evaluate the suitability of the new energy storage system, in terms of hydrogen consumption and achievable riding range.

\section{Power unit design}

The original e-bike is powered by a 250W@36V brushless electric motor (EM) mounted on the rear wheel, and it has a battery pack with capacity of $10 \mathrm{Ah}(360 \mathrm{Wh})$. The new HyBike keeps the same EM and driver, but its hybrid power unit is composed instead by a PEM fuel cell

*Corresponding author: paolo.digiorgio@uniparthenope.it 
(FC) and a smaller battery pack. The hydrogen is solidstate stored in a MH tank that operates also as thermal management system for the battery pack. The FC is connected to the battery pack via a DC/DC step-up converter, that stabilizes the output voltage of the $\mathrm{FC}$ and controls its power output. In this way, the power produced by the FC stack can be either used by the EM or to charge the battery pack. A schematic representation of the fuel cell/battery hybrid power unit architecture is reported in Fig. 1.

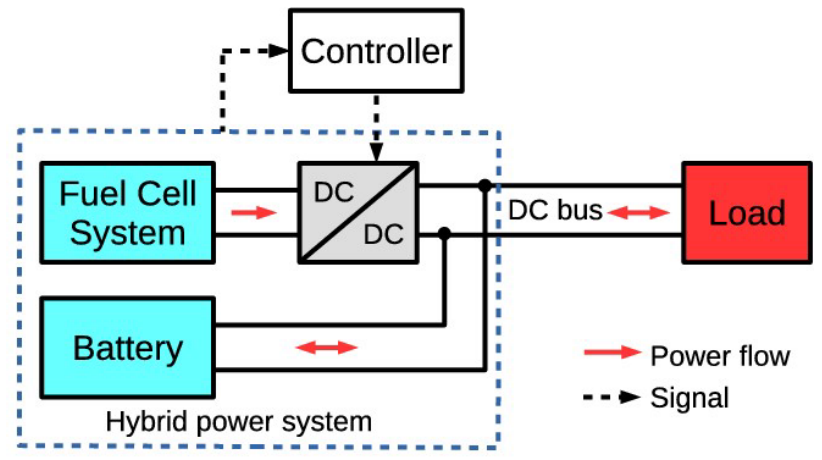

Fig. 1. Schematic of the HyBike power unit architecture.

The battery in the new power unit provides the power required by the EM during transient operations, while the FC operates basically as a range extender, providing the required average power and avoiding the battery pack State of Charge (SoC) to drop below a minimum threshold. In particular, the FC charges the battery whenever the power requested by the EM is sufficiently low, while the battery is discharged when the power demand is higher than the power output of the FC. Anyhow, the charging current of the battery pack can never be higher than its allowed maximum (that for a lithium-ion battery is usually much lower than the discharge current one).

\subsection{Data acquisition}

The design of power unit components was performed starting from the power profile acquired during the normal operation of the vehicle. To this aim, a large onroad data acquisition campaign has been carried out with the original un-modified model of the e-bike, powered by the $10 \mathrm{Ah}, 36 \mathrm{~V}$ battery pack. Both battery voltage and current profiles were collected for 25-30 minutes for each test. As an illustrative example, two EM power profiles are shown in Fig. 2, which are representative of two different vehicle operative conditions: panel (a) refers to a typical urban use, hereafter referred as Urban Cycle A, with not frequent uphill and downhill, and characterized by several stops; panel (b) refers instead to a more severe path, hereafter referred as Urban Cycle B, presenting several climbs, which is consequently a more power demanding scenario. Both paths were about $7.5 \mathrm{~km}$ long. The duty cycle analysis revealed that, despite the nominal maximum continuous power of the electrical motor being $250 \mathrm{~W}$, its measured power output reaches above $600 \mathrm{~W}$ during transient operations.
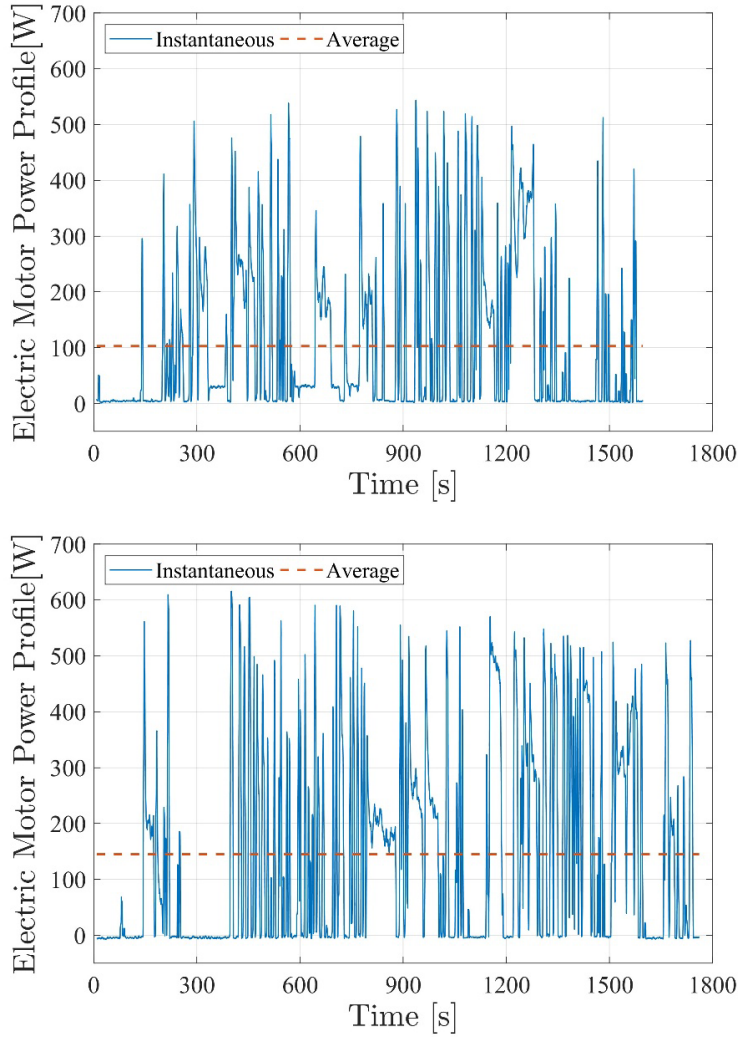

Fig. 2. EM power demand acquired during two road-tests. Top: Urban Cycle A. Bottom: Urban Cycle B.

For the two cases shown in Fig. 2, the average power consumption is equal to $103 \mathrm{~W}$ and $145 \mathrm{~W}$, for Urban Cycle A and Urban Cycle B conditions, respectively, while the total energy requested by the EM amounts to 45 Wh and $71 \mathrm{Wh}$, respectively.

\subsection{Fuel cell module}

The size of the FC was determined basing on the maximum average power consumption of the EM acquired during the tests. The Horizon FCS-C200 PEM Fuel Cell [12], with a rated power of $200 \mathrm{~W}$, was the final choice, since this model is able to provide the reference average power, and given also that its dimensions fit well with the available space on-board of the bicycle. Thus, this FC was bench-tested in order to characterize its performances.

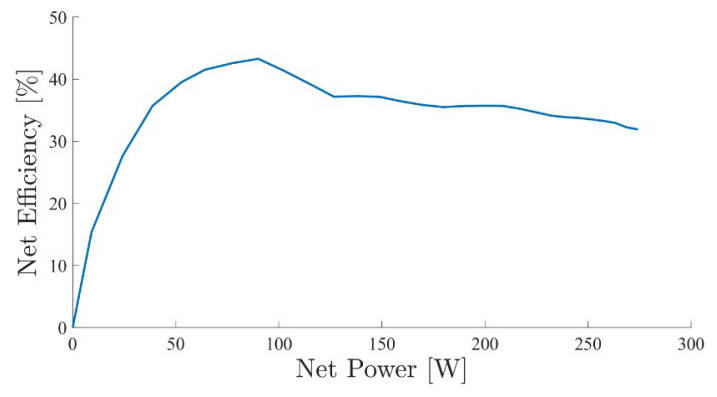

Fig. 3. Fuel cell efficiency curve. 
Fig. 3 shows the measured FC net efficiency against the net power output. It is interesting to note how the peak efficiency is reached at about $100 \mathrm{~W}$, while it decreases significantly for higher and, especially, lower values of power.

The power of the $\mathrm{FC}$ is controlled by a DC/DC converter placed between the FC and the battery pack. Since a programmable DC/DC converter that is suitable for the specific application was not find available on the market, a commercial DC/DC converter was instead used after ad-hoc modified. This is shown in Fig. 4.

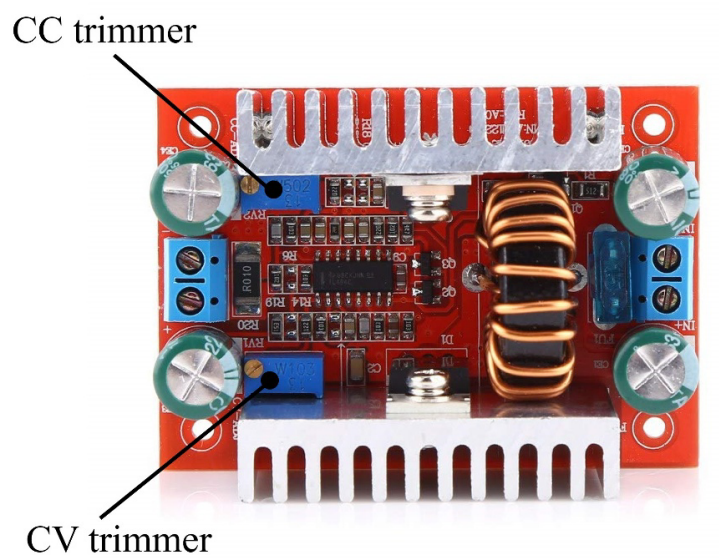

Fig. 4. The employed DC/DC converter, before the trimmer substitution.

The used converter is able to work both in $\mathrm{CV}$ mode (constant output voltage) and CC mode (constant output current): when the current is lower than the reference value (set through the $\mathrm{CC}$ trimmer), the $\mathrm{DC} / \mathrm{DC}$ converter provides a constant output voltage (set through the $\mathrm{CV}$ trimmer); otherwise, as the current tends to exceed the reference value, the output voltage is lowered in order to maintain the output current equal to the reference value. Substituting the $\mathrm{CC}$ trimmer with a digital potentiometer, which is a variable resistance controlled by an electronic control unit, it is possible to control in real-time the DC/DC output current and so the power supplied by the FC. The CV trimmer was set to an output voltage of 41 $\mathrm{V}$, so that the battery overcharging would be avoided in any case (the maximum operating voltage for a $36 \mathrm{~V}$ battery pack is $42 \mathrm{~V}$ ).

The power unit of the HyBike must be contained in a small space and arranged in such a way as not to negatively affect the comfort and drivability of the vehicle. Therefore, an extremely compact design was realized for the casing containing the power unit, which was finally positioned at the rear of the HyBike, as shown in Fig. 5.

\subsection{Battery pack}

The battery cells used for the new battery pack are lithium Nickel-Cobalt-Aluminum oxyde (NCA) cells provided by GWL, model LG MH1 [13], having nominal voltage of $3.67 \mathrm{~V}$ and maximum discharge current of 10 A. Their main features are summarized in Table 1 .

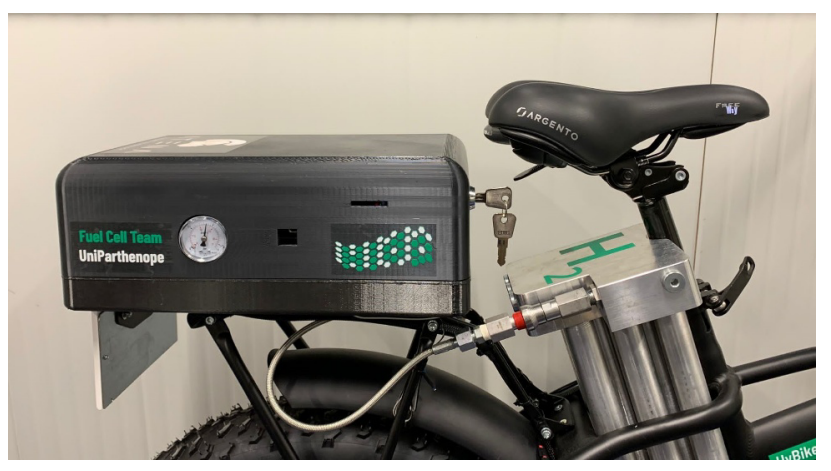

Fig. 5. Casing of the HyBike power unit.

The battery pack is formed by two modules connected in series, each having a $5 \mathrm{~s} 2 \mathrm{p}$ configuration (Fig. 6-a). This allows to reach the nominal voltage of the EM and to provide the required maximum discharge current measured during the data acquisition (about $20 \mathrm{~A}$, corresponding to roughly $700 \mathrm{~W}$ of discharge power). The total capacity of the battery pack is $6.4 \mathrm{Ah}(230 \mathrm{Wh})$, that corresponds to about $65 \%$ of the capacity of the battery pack powering the original e-bike.

Table 1. Main characteristics of the LG MH1 battery cells used in the HyBike.

\begin{tabular}{ll}
\hline Model & LG MH1 \\
Chemistry & NCA \\
Nominal Voltage [V] & 3.67 \\
Nominal Capacity [Ah] & 3.2 \\
Max continuous discharge current [A] & $10(3.125 \mathrm{C})$ \\
Max continuous charge current [A] & $3.2(1.0 \mathrm{C})$ \\
Recommended charge current [A] & $1.6(0.5 \mathrm{C})$ \\
\hline
\end{tabular}

\subsection{Power sharing algorithm}

In order to prevent malfunctioning and damages of the battery pack, its charging current should not exceed the $3.2 \mathrm{~A}(0.5 \mathrm{C})$, which corresponds to about $100 \mathrm{~W}$. On the other hand, in order to avoid an excessive depletion of the battery SoC, the FC mean power output should be at least equal to the mean power required by the EM during vehicle operation (i.e. about $145 \mathrm{~W}$, according to data acquisition). These considerations pose a constraint to the choice of a suitable energy management strategy. Therefore, the power split between FC and battery is setup according to a rule-based control strategy, with a feedback control on battery SoC, which distinguishes three modes of operation, as follows:

- $\quad$ Charge Depleting (CD): for a battery SoC higher than $70 \%$, the HyBike run as a BEV.

- $\quad$ Charge Sustaining - constant power (CS-CP): the first time the battery SoC goes below $70 \%$, or anytime the battery SoC hits the $60 \%$, the FC is activated so to provide constant power at $100 \mathrm{~W}$ to support battery operation and/or partially recharge the battery, whenever the power request from the EM is lower than the FC power. This mode of operation is held until the SoC reaches a lower threshold value set equal to $50 \%$. 
- $\quad$ Charge Sustaining - load following (CS-LF): as the battery $\mathrm{SoC}$ reaches/is below its lower threshold $(50 \%)$, the FC starts to operate by following the EM request and possibly recharging the battery, that is, according to a load following mode. Therefore, in this case, the FC power output is not constant anymore, but rather it is equal to the (variable) power requested by the EM plus a base constant load of 100 W. Under this mode of operation, the battery is used only to compensate the request of power whenever this is higher than that available from the FC, which is bounded to its allowed maximum. This approach allows for an optimal recharging strategy of the battery.

In order to ensure the proper functioning of the $\mathrm{FC}$, a maximum power ramp rate given by $1.5 \mathrm{~A} / \mathrm{s}$ has been imposed. The CS-CP and CS-LF modes of operation described above are alternatively activated so to perform a hysteresis cycle between the two predefined battery SoC limits.

\section{Integrated energy storage system}

The energy storage of the HyBike is a combined system where the battery pack and a $\mathrm{MH}$ tank are thermally integrated to each other into a compact device. By this design, an optimal self-sustained thermal management of the two components is realized, since the heat produced by the battery during operation is efficiently removed and transferred to the $\mathrm{MH}$ tank, where an endothermic hydrogen desorption process occurs.

In general, the choice for the most suitable alloy to be used as hydrogen storage material in MHs depends on the system operating conditions. Therefore, the hydride material chosen for the MH tank of the HyBike was selected among the commercially available ambienttemperature $\left(0-30^{\circ} \mathrm{C}\right)$ alloys. Also, the maximum recharge pressure of the $\mathrm{MH}$ tank was set to $20 \mathrm{bar}$, in order to contain the wall thickness and the overall weight. This value for the pressure is compatible with the FC

hydrogen feeding pressure, that is around $0.45-0.55$ barg according to the FC technical data sheet. In addition to good absorption-desorption properties within the operating temperature and pressure conditions, the selected alloy should also i) be easy to activate, ii) have fast kinetics, iii) have high reversible gravimetric capacity, and iv) have good cyclability. Among all the intermetallic compounds responding to these requirements [14-16], the Hydralloy $C 5^{\circledR}$ supplied by Gesellsclhaft für Elektrometallurgie (GfE) was finally selected [17].

The MH tank of the HyBike is composed of eight aluminium cylinders, two of which are integrated with the battery cells to form a hexagonal modular structure, while the remaining six are external to the battery pack and exposed to air. Pictures for the prototypes of $\mathrm{MH}$ tank and assembled hybrid energy storage system are shown in Fig. 6. The cylindrical battery cells and the two integrated $\mathrm{MH}$ canisters are allocated in a $3 \mathrm{D}$ printed holder made of conductive PLA (GRAPHYLON3D) with $100 \%$ filling, which promotes the heat exchange between the two components. In particular, the size of the cylinders containing the $\mathrm{MH}$ was determined upon the estimation of the heat generated by the battery cells that has to be removed. This calculation led to an internal diameter of $24 \mathrm{~mm}$ for all the cylinders, and to a length of $450 \mathrm{~mm}$ for the 2 integrated cylinders, while the remaining 6 have a length of $250 \mathrm{~mm}$. The external diameter of all cylinders was set to $30 \mathrm{~mm}$. The final hydrogen capability of the MH tank was $50 \mathrm{~g}$, for an overall weight of about $7 \mathrm{~kg}$.

All the cylinders are externally threaded on one end (M30 x 1.5) so that they can be screwed-in a flange, which connect them together. The hydrogen flows through a $1 \mu \mathrm{m}$ Swagelock filter before reaching a pressure reducer and then the FC stack. The sealing between the flange and the cylinders is guaranteed by a Teflon gasket. On the opposite end the cylinders are internally threaded and closed by an end cup with o-ring. The battery pack is enclosed in a carter and connected to the bicycle frame using screws, while the MH tank can be easily removed to be refilled.

(b)

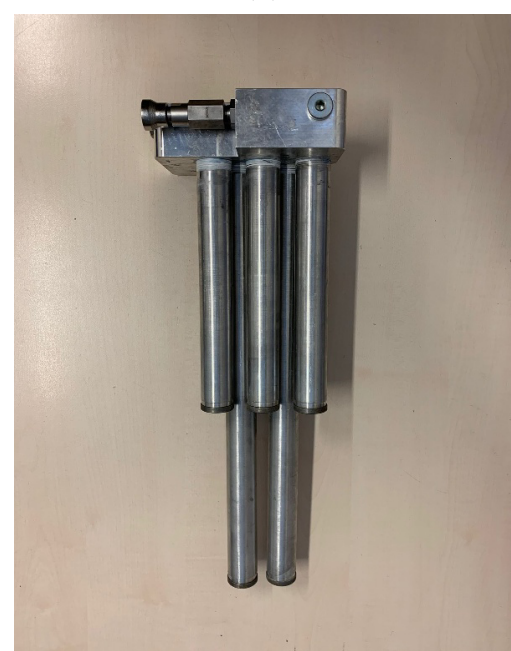

(c)

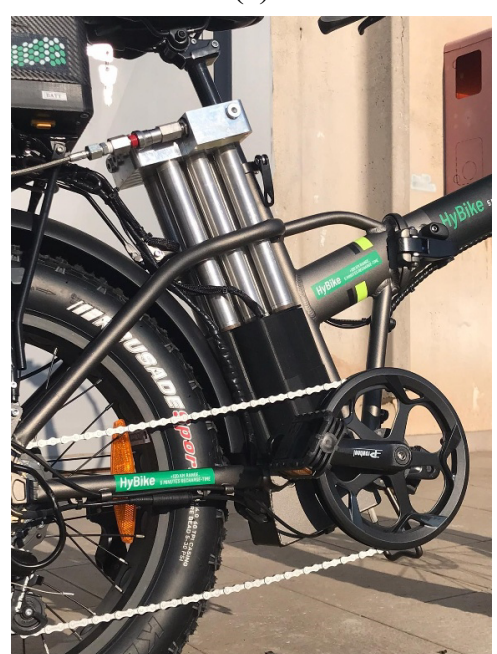

Fig. 6. Hybrid energy storage system of the HyBike: a) single module of the battery pack, b) MH tank, c) assembling of the storage system on the HyBike prototype. 


\section{Experimental results}

In order to verify the correct operation of the designed system, two experimental tests were carried out. These were performed by using a regenerative BTS, which uses the power profiles acquired during the design phase (Fig. 2) to emulate the actual vehicle operation. The employed BTS is the IT8005-80-150 model, produced by ITECH, which has maximum power, voltage and current output equal to $5 \mathrm{~kW}, 80 \mathrm{~V}$ and $150 \mathrm{~A}$, respectively.

Specifically, with the aim of investigating the power unit performances under the different modes of operation prescribed by the power sharing control algorithm, the two tests were carried out by imposing different initial conditions, in terms of battery SoC: for the Urban Cycle A, the initial battery SoC has been set to $39 \%$, while for the Urban Cycle B, an initial value of $67 \%$ has been chosen. The obtained battery SoC profiles, during the two tests, are shown in Fig. 7.
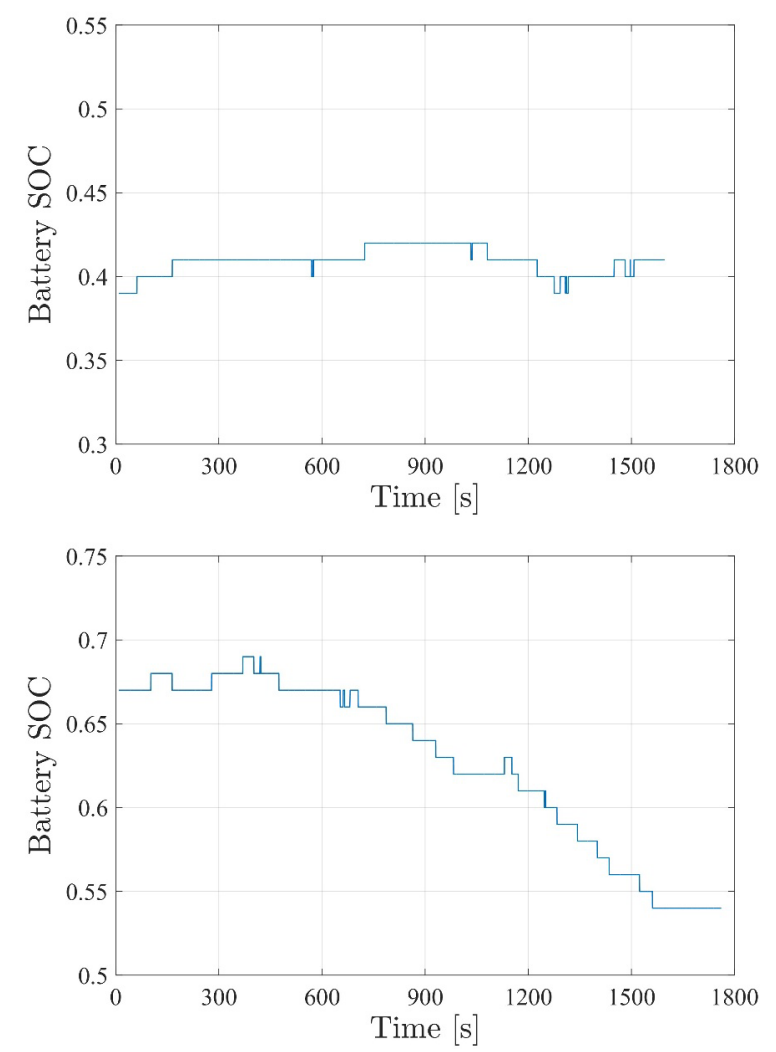

Fig. 7. Battery SoC profiles recorded during the two experiments. Top: Urban Cycle A. Bottom: Urban Cycle B.

The battery SoC remains fairly constant and always below 50\% during the Urban Cycle A: in this case, the FC operates according to a CS-LF mode. In contrast, the battery SoC decreases during the Urban Cycle B, finally reaching a value of a $54 \%$ : in this case, the CS-CP mode of operation is the activated mode. Next, Fig. 8 reports the obtained power output profiles recorded during the two experimental tests. The results show that the power unit is able to provide the power requested by the EM during both the analyzed scenarios and under the two different power sharing modes, while preserving at the same time the correct operation of its components.
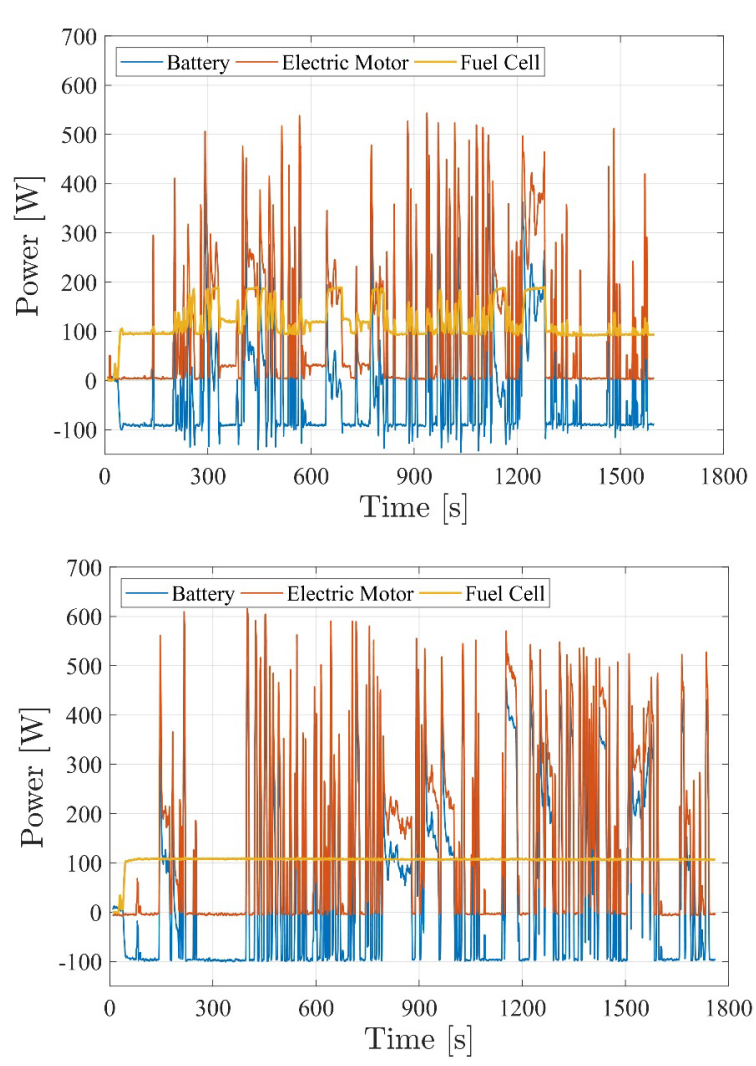

Fig. 8. Obtained power profile for FC and battery vs EM power demand. Top: Urban Cycle A. Bottom: Urban Cycle B.

In Table 2 the evaluated FC average efficiency and the measured hydrogen consumption are reported.

Table 2. Fuel cell performance and hydrogen consumption during experimental tests.

\begin{tabular}{l|cc} 
& $\begin{array}{c}\text { Average FC } \\
\text { efficiency [\%] }\end{array}$ & $\begin{array}{c}\mathrm{H}_{2} \text { consumption } \\
{[\mathrm{g}]}\end{array}$ \\
\hline Urban Cycle A & 36 & 4.4 \\
Urban Cycle B & 42 & 3.8 \\
\hline
\end{tabular}

It should be noted that the average $\mathrm{FC}$ efficiency is higher for the Urban Cycle B since, in this case, the FC operates at constant power, with value close to the point of maximum efficiency (see Fig. 3). Considering that the $\mathrm{MH}$ storage tank has a hydrogen capacity of $50 \mathrm{~g}$, the HyBike results to achieve a riding range of about $90 \mathrm{~km}$ in charge sustaining mode, while its all electric range, assuming a battery Depth of Discharge (DoD) of $80 \%$, is about $25 \mathrm{~km}$, thus leading to an overall estimated range of $115 \mathrm{~km}$. This value is significantly higher than that for the original e-bike, for which a riding range of roughly 40 $\mathrm{km}$ is estimated, considering the same test conditions and under the same assumption of battery DoD.

\section{Conclusions}

A new hydrogen-powered bicycle has been presented in this study. The peculiarity of this vehicle lies in its onboard energy storage system: a hybrid system which is based on the thermal integration between a metal hydride 
tank and the battery pack. By this way, not only the storage energy density of the vehicle is enhanced, but also an optimal thermal management of the two components may be achieved.

Specifically, the design of the power unit of the vehicle and of its hybrid energy storage system have been shown in this work. Thus, a prototype has been realized and bench-tested, in order to check design consistency and to evaluate its performances. The results are very promising: the riding range of the HyBike is about three times higher the one of the original e-bike. This may open interesting prospects towards a more sustainable urban mobility, since the new HyBike can potentially play a significant role in the light-vehicles panorama. Further investigations will be devoted to the assessment of the hybrid energy storage system, in terms of thermal management capabilities.

This research was funded by the project HyLIVE - Hydrogen Light Innovative Vehicles, grant n. B63D18000430007, under the program POR Campania FESR 2014/2020.

\section{References}

1. G. G. Nassif and S. C. A. d. Almeida, Int. J. Hydrogen Energy 45, 21722 (2020)

2. H. Zhang, X. Li, X. Liu, and J. Yan, Appl. Energy 241, 483 (2019)

3. Fuel Cells Bull. 2018, 4 (2018)

4. G. Di Ilio, P. Di Giorgio, L. Tribioli, G. Bella, and E. Jannelli, Energy Convers. Manag. 243, $114423(2021)$

5. G. Di Ilio, P. Di Giorgio, L. Tribioli, V. Cigolotti, G. Bella, and E. Jannelli, SAE Tech. Pap. 202124-0109 (2021)

6. M. Minutillo, A. Forcina, N. Jannelli, and A. Lubrano Lavadera, Energy 153, 200 (2018)

7. J. L. Shang and B. G. Pollet, Int. J. Hydrogen Energy 35, 12709 (2010)

8. P. Di Trolio, P. Di Giorgio, M. Genovese, E. Frasci, and M. Minutillo, Appl. Energy 279, 115734 (2020)

9. A. Perna, M. Minutillo, S. Di Micco, V. Cigolotti, and A. Pianese, E3S Web Conf. 197, 5001 (2020)

10. C. Zamfirescu and I. Dincer, J. Power Sources 185, 459 (2008)

11. K. P. Brooks, S. J. Sprik, D. A. Tamburello, and M. J. Thornton, Int. J. Hydrogen Energy 45, 24917 (2020)

12. Horizon Fuel Cell Technologies, $H$-200 Fuel Cell Stack User Manual (2021)

13. GWL Power, Li-Ion Cell LG MH1 Data Sheet (2021)

14. B. Tanç, H. T. Arat, Ç. Conker, E. Baltacioğlu, and K. Aydin, Int. J. Hydrogen Energy 45, 26344 (2019)

15. C. H. Chao and J. J. Shieh, Int. J. Hydrogen Energy 37, 13141 (2012)

16. L. Xu, M. Ouyang, J. Li, F. Yang, L. Lu, and J. Hua, Appl. Energy 103, 477 (2013)
17. B. Sakintuna, F. Lamari-Darkrim, and $M$. Hirscher, Int. J. Hydrogen Energy 32, 1121 (2007) 